\title{
Can the world be shown to be indeterministic after all?
}

\author{
Christian Wüthrich
}

\begin{abstract}
This essay considers and evaluates recent results and arguments from classical chaotic systems theory and non-relativistic quantum mechanics that pertain to the question of whether our world is deterministic or indeterministic. While the classical results are inconclusive, quantum mechanics is often assumed to establish indeterminism insofar as the measurement process involves an ineliminable stochastic element, even though the dynamics between two measurements is considered fully deterministic. While this latter claim concerning the Schrödinger evolution must be qualified, the former fully depends on a resolution of the measurement problem. Two alleged proofs that nature is indeterministic, relying, in turn, on Gleason's theorem and Conway and Kochen's recent 'free will theorem', are shown to be wanting qua proofs of indeterminism. We are thus left with the conclusion that the determinism question remains open.
\end{abstract}

Do probabilities exist out there in the world, independently of our epistemic situation in it; or are they mere indications of our imperfect knowledge of matters of fact? This old question has recently, and very recently, been enriched by new results. The purpose of this paper is to introduce and discuss these results, as well as to appraise the status of probabilities as objective or subjective in a world that is either deterministic or indeterministic. But first and foremost, this essay addresses the dual issues of whether our world is deterministic or indeterministic and of whether we can ever know this.

There are at least two roles that probabilities can play in a dynamical theory. First, they may codify a distribution over initial or boundary conditions. Second, probabilities may concern the dynamical evolution given a certain initial state of the physical system at stake. Orthogonally, probabilities in either role may be objectively in the world, i.e. real world chances, or they may be subjective and arise only due to our ignorance of the exact state of affairs in the world. A subjective probability distribution over initial conditions would simply mean that although it is the case that the world was in some particular initial state, we don't know which and the probability distribution encodes our best guess concerning the likelihoods with which the different initial states obtained. It is somewhat less straightforward to say what objective probabilities on initial conditions might be. They may be generated by a random process, but as we turn to the initial conditions of the whole universe, this approach no longer succeeds. One way to get them may be via a multiverse, perhaps of non-denumerably many universes, where the probabilities over initial states capture the (measure-theoretic) frequency of the particular initial states. Note that in this case, we may have objective and subjective probabilities, where the latter need not coincide with the objective ones, but rather result from our limited knowledge about the objective distribution. Another way, following Barry Loewer (2001), would be to argue that a Humean best-system analysis entails that the probability distribution over initial states in statistical mechanics is objective.

For the probabilities pertaining to the dynamical evolution, the dynamical probabilities, the existence, at the fundamental level, of objective and subjective probabilities seems to line up neatly with indeterministic and deterministic worlds, respectively. If the world is deterministic, then one would expect that the (non-trivial) dynamical probabilities that feature in physical theories are nothing but reflections of our incomplete knowledge of the dynamics in that world. Objective 
probabilities, if they exist, would either only arise at a higher, derivative level resulting from some form of course-graining or time-averaging, or else from a Humean interpretation of probability distributions over initial conditions (see Maudlin's chapter). On this understanding, objective probabilities in a deterministic world would either not be fundamental or not dynamical. On the other hand, if the world is indeterministic, a complete dynamical theory of it would have to incorporate probabilities to account for the indeterministic evolution. ${ }^{1}$ In this case, one could rightfully say that the probabilities are 'in the world' and hence objective.

In this essay, I shall mainly be concerned with dynamical probabilities, rather than probability distributions over initial conditions. However, it should be noted that the close association of fundamental objective dynamical probabilities with indeterminism only holds if a principled distinction between initial conditions and the dynamical laws can be maintained. If this distinction crumbles, and there seem respectable reasons to think that it could, then the Humean move in Loewer 2001 will invest even a deterministic world with non-trivial fundamental objective probabilities. That the determination of whether there exist fundamental objective probabilities in a deterministic world seems to hinge on the distinction between initial conditions and dynamical laws is extremely interesting, but shall be pursued on another day.

Thus, if we wish to know whether the probabilities in physics, as they pertain to dynamics, are objective or subjective, we need to inquire into whether the world is deterministic or indeterministic. From the naturalistic point of view that is assumed in this essay, this metaphysical question deflates into the question of whether our best physical theories entail that the world is deterministic or indeterministic. I will not offer a comprehensive survey of the fate of determinism in physical theories; the interested reader is referred to the systematic, and magisterial, treatment of this issue by John Earman, in particular in Earman 1986, 2004, and 2007 2 Finally, there is the further, 'transcendental,' question of whether we can ever come to know, in principle, whether the world is deterministic or indeterministic.

After having set up the issue in Section 1, and some brief remarks concerning classical chaotic systems, I will move on to address the leading question in the context of quantum mechanics. Section 2 explicates how in non-relativistic quantum mechanics the issue hangs not only on the solution of the measurement problem, but even on a proper understanding of mundane Schrödinger dynamics. It also returns to transcendence by investigating whether the empirical equivalence of Bohmian mechanics and Nelson's mechanics establishes that determinism transcends any empirical accessibility. Section 3 discusses whether arguments for indeterminism based on Gleason's theorem and the so-called 'free will theorem,' respectively, succeed. This will be answered in the negative for both cases. Finally, Section 4 offers conclusions.

\section{Setting up the transcendental question, and addressing it}

Is the world, in a sense yet to be specified, deterministic or indeterministic? And, whichever is the case, can we ever know this? Immanuel Kant, in the Third Antinomy of the Transcendental Dialectic of his Critique of Pure Reason, addresses these questions. Kant's interest in the issue is grounded in the problem of the freedom of human will, which, in his view, requires at least some degree of genuine indeterminism in order to have a fighting chance. An antinomy, for Kant, is a pair of contradicting claims - the 'Thesis' and the 'Antithesis' - that both muster equal rational

\footnotetext{
${ }^{1}$ The qualification of completeness is important to rule out potential counterexamples such as Norton's dome (2008), where no probabilities are present. In cases like this, the theory on offer is arguably incomplete as it remains silent concerning probability distributions over possible evolutions.

${ }^{2}$ Cf. also Hoefer (2003, Sec. 4).
} 
support, at least initially. In the case of the Third Antinomy, the Thesis holds that

Causality, according to the laws of nature, is not the only causality from which all the phenomena of the world can be deduced. In order to account for these phenomena it is necessary also to admit another causality, that of freedom. (A444)

On the other hand, the Antithesis maintains that

There is no freedom, but everything in the world takes place entirely according to the laws of nature. (A445)

Kant provided and examined a priori arguments for both Thesis and Antithesis, where on a straightforward reading the former claims indeterminism and the latter asserts determinism. Without going into the intricacies of Kant's arguments for either of these theses or for his own resolution of the antinomy, we can ask, with Patrick Suppes (1993), whether either or both theses could be supported empirically. Before this issue can be broached, let me fix the meaning of determinism. I will use it in the Laplacean sense as defined in Earman 1986 (Ch. 2). Although the existence of a cause for each effect or the type identity of effects given the type identity of their causes have variously been called the 'principle of determinism,' I shall leave the thicket of causation to one side and operate with a conceptualization of determinism that is entirely devoid of causal language. Similarly, I wish to keep determinism and predictability conceptually separate. Although this will not be argued here, there are good reasons for this separation 3

Following Earman $(1986,13)$, let then $\mathcal{W}$ denote the set of all physically possible worlds, i.e. those possible worlds which are in accordance with the laws of the actual world. For worlds, then, we can introduce the following notion of determinism:

Definition 1 (Determinism for worlds) $A$ world $W \in \mathcal{W}$ is deterministic if and only if for any $W^{\prime} \in \mathcal{W}$, if $W$ and $W^{\prime}$ agree at any time, then they agree for all times.

A world that fails to be deterministic will be called indeterministic. In the naturalistic vein presupposed in this essay, we will reinterpret the question of whether our world is deterministic as asking what answers our scientific theories entail. The following definition naturally extends the meaning of determinism to theories:

Definition 2 (Determinism for theories) $A$ 'theory $T$ is deterministic just in case, given the state description $s\left(t_{1}\right)$ at any time $t_{1}$, the state description $s\left(t_{2}\right)$ at any other time $t_{2}$ is deducible [in principle] from T.' (ibid., 20)

A theory that fails to be deterministic is said to be indeterministic. Equipped with these definitions, let us return to the transcendental question.

Analyzing dynamical systems, and classical chaos in particular, Suppes (1993) concludes that the question of whether our world is deterministic or indeterministic transcends, in fact must transcend, any possible experience since Thesis and Antithesis are equally validated by empirical evidence. Suppose we are studying a particular physical process for which it is unknown whether its dynamics is deterministic or indeterministic. A series of observations - such as measurements of the position(s) at particular times - is made and our task is then to determine whether, given the data, the process is best described as deterministic or indeterministic. Suppes argues that deterministic and indeterministic descriptions can equally be given. He bases this claim on a result

\footnotetext{
${ }^{3}$ Cf. e.g. Earman (1986, Secs. 2.3-2.4).
} 
known as Ornstein's theorem, which, in his words, states that there are 'processes which can equally well be analyzed as deterministic systems of classical mechanics or as indeterministic semi-Markov processes, no matter how many observations are made' (254). He also assumes that this theorem applies to 'most physical processes above a certain complexity level.' This suggests that, he thinks, '[f]or a great variety of empirical phenomena there is no clear scientific way of deciding whether the appropriate "ultimate" theory should be deterministic or indeterministic' (ibid.).

Apart from a brief foray into quantum mechanics, where he denies that quantum mechanics by itself offers a conclusive argument in favour of indeterminism - a view for which I have sympathies, the entire essay deals with classical physics. However, the 'ultimate' theory, if there is one, will not be a classical but a quantum theory. Since it can be shown that there is no simple relation to be had between how determinism fares in classical and quantum physics $4^{4}$ addressing the issue purely in classical physics is going to reveal precious little about what judgments we will reach when considering the problem in the 'ultimate' quantum theory. In the light of this, the confinement to classical theories is a serious lacuna in Suppes's argument.

Furthermore, Suppes's claim does not even seem to succeed for classical chaotic systems. While it seems true that for a given sequence of position measurements with a certain finite accuracy, one can always concoct a deterministic evolution that generates it as well as describe it as a stochastic, and hence indeterministic, process, this in itself does not imply that chaotic deterministic and genuinely stochastic models are empirically indistinguishable. In fact, as John Winnie (1997) has shown, neither Ornstein's theorem nor related results support such an inference. At heart, Winnie explains (317), the issue is that there is an asymmetry between the deterministic and the stochastic description, which comes out under the assumption that for any particular coarsegrained measurement scale, one can always make more accurate measurements, at least in principle 5 While it is typically possible to generate a discrete-time stochastic process from the appropriately partitioned underlying continuous-time deterministic process, the converse is not true: a complete deterministic description of a process contains information on the system's behaviour at scales below the coarse-graining scale, information which a stochastic description lacks. From this, Winnie concludes that the deterministic description is conceptually prior to the coarse-grained random dynamics. Thus, the philosophical gloss of Ornstein's theorem offered by Suppes does not hold water and does not establish that the Thesis and the Antithesis can summon equal empirical support.6 With that, let us turn to quantum mechanics.

\footnotetext{
${ }^{4}$ Cf. Earman 2008 and Wüthrich 2006 (Ch. 6).

${ }^{5}$ Winnie (318) believes that this assumption is borne out in classical physics, where we have no principled limitations to observational accuracy.

${ }^{6}$ Werndl (2009, and in private communication) begs to differ. If we do not know, she claims, whether a deterministic or stochastic description is correct we also don't know whether there are in principle limitations of our observational accuracy. There may well be such limitations and with them, Winnie's asymmetry may not come into play. She concludes that, at least for a vast class of physical systems, deterministic and stochastic descriptions are empirically indistinguishable. But apart from the fact that in classical physics, there seem to be no grounds on which to believe in such principled limitations, the system at stake either is, in actuality, deterministic or stochastic. If the former, considerations along Winnie's line suggest that the descriptive poverty of the stochastic process vis-à-vis the deterministic competitor may show in an empirically accessible manner. This seems to imply that at least in a deterministic world, the competing descriptions are not empirically indistinguishable. Of course I agree with her that which description is preferable depends on a number of factors, such as the phenomena under consideration and the theoretical framework within which we tackle them.
} 


\section{Indeterminism in quantum mechanics}

Quantum mechanics, it is often claimed, is an ineliminably indeterministic theory. Quantummechanical phenomena such as radioactive decay or the absorption or emission of photons, it is widely believed, can only be described in a probabilistic fashion. In this sense, quantum mechanics is usually held to establish that there are objective probabilities in the actual world. If it were unqualifiedly true that quantum mechanics is indeterministic 7 then the transcendence claim would be false and probabilities would objectively and ineliminably be part of our world. However, the issue whether quantum mechanics is indeed indeterministic is much subtler and richer than is sometimes appreciated.

Arguably, the reason why most physicists and many philosophers believe that quantum mechanics is inherently indeterministic is because the formerly standard Copenhagen 'interpretation' includes John von Neumann's Projection Postulate, which stipulates a stochastic 'collapse' of the wave function upon an interaction of the system with a 'measurement apparatus.' It is widely agreed, however, that between these 'measurement interactions,' a quantum-mechanical system evolves deterministically in accordance with Schrödinger's equation, the fundamental dynamical law of non-relativistic quantum mechanics. In spite of their popularity, both these claims are misleading at best and simply false at worst. Whether or not a solution of the measurement problem requires an indeterministic theory is wide open. But first, let me address, and thereby qualify, the claim that the Schrödinger evolution is deterministic.

\subsection{Reconsidering the Schrödinger evolution}

The Schrödinger equation is a first-order, linear partial differential equation for which there are existence and uniqueness theorems. The caveat with these theorems, however, is that they have certain antecedent conditions; in particular, they impose some conditions on the form the Hamiltonian can take. As John Norton (1999) has argued, the Schrödinger evolution can become indeterministic under the rather extreme conditions of a 'quantum supertask.' The reason for this is that under these conditions, the differential form of the Schrödinger equation is not equivalent to its integral form and it is the latter form for which the theorems assure the existence of a unitary time-evolution operator of the form $\hat{U}(t)=\exp (-i \hat{H} t)$ guaranteeing a unique, and hence deterministic, evolution. In the pathological cases studied by Norton, the evolution can be rendered deterministic again if an additional constraint is imposed on the state vector.

Somewhat orthogonally, as Earman (2009) has very recently urged, those instances when the dynamics of a system is governed by a non-essentially self-adjoint Hamiltonian ought to be regarded as indeterministic. What is a 'non-essentially self-adjoint' Hamiltonian? In order for a candidate Hamiltonian to qualify as a genuine operator, its domain of definition must be specified. This is necessary because, typically, these candidates are operators defined only on a dense domain of the Hilbert space but not generally on all of it. The task, then, is to find, if possible, selfadjoint extensions of those operators to the entire Hilbert space. Usually, there is an obvious choice of a dense domain on which the candidate Hamiltonian $\hat{H}$ is self-adjoint 8 The task then is to extend $\hat{H}$ onto the remainder of the Hilbert space such that this extended operator is selfadjoint. Sometimes $\hat{H}$ does not have a unique self-adjoint extension. If it does, $\hat{H}$ is said to be essentially self-adjoint. It is usually assumed in physical applications that $\hat{H}$ is essentially self-

\footnotetext{
${ }^{7}$....and if the fundamental or ultimate theory is quantum-mechanical in every respect relevant to the issue of whether it is deterministic or not...

${ }^{8}$ An operator $\hat{O}$ that is densely defined on a Hilbert space $\mathcal{H}$ is self-adjoint iff $\hat{O}=\hat{O}^{*}$, where ${ }^{*}$ denotes the adjoint. Self-adjoint operators are important because observables are standardly represented by self-adjoint operators.
} 
adjoint. However, Earman (2009) points to a number of examples where the Hamiltonian is not essentially self-adjoint. Although it is certainly a question that philosophers of physics ought to tackle systematically, I will not here delve into whether the Hamiltonian is essentially self-adjoint in all physically possible situations. It suffices to point out that this is largely open and that the essential self-adjointness of the Hamiltonian of a system is thus, at least until further notice, a substantive assumption that is made - often tacitly.

But why should we follow Earman (2009) in equating the failure of essential self-adjointness of the Hamiltonian with a failure of determinism of the usual Schrödinger evolution? Suppose we study a physical system whose Hamiltonian $\hat{H}$ is not essentially self-adjoint, i.e. there exist several distinct self-adjoint extensions $\hat{H}_{1}^{\prime}, \hat{H}_{2}^{\prime}, \ldots$ of $\hat{H}$. Assuming that the quantum dynamics results from a continuous unitary group of operators $\hat{U}(t)$, the distinct extensions generate physically distinct dynamical evolutions by leading to distinct one-parameter unitary groups $\hat{U}_{1}^{\prime}(t):=\exp \left(-i \hat{H}_{1}^{\prime} t\right), \hat{U}_{2}^{\prime}(t):=\exp \left(-i \hat{H}_{2}^{\prime} t\right), \ldots$ governing the evolution. Technical details aside, what this means is that a given state at some initial time will, in general, evolve differently for later times depending on which extension is chosen. The form of indeterminism that emerges is, of course, quite different from the known forms of indeterminism arising in classical physics or state vector reduction.

According to Definition 2, the dynamical theory of this system is not deterministic unless the theory has the resources to deal with this non-uniqueness. Earman recognizes two ways in which this could be done: either stipulate one of the self-adjoint extensions as being the one generating the true physical evolution, or suppress any Hamiltonian that is not essentially selfadjoint as physically impossible. The latter move renders determinism necessarily true in noncollapse quantum mechanics. Earman rightly rejects such 'high-handedness,' in particular in the light of the possibility that the quantum counterpart of an actually existing classical system may have a non-essentially self-adjoint Hamiltonian. Earman also finds the former strategy unattractive since the uniqueness of the temporal evolution 'was supposed to flow from the laws of motion themselves' (2009, 36), which would no longer be the case as new physical principles would have to be added to the laws of motion in order to guarantee the uniqueness of the evolution.

The choice among distinct self-adjoint extensions of the Hamiltonian amounts to a choice among distinct conditions at the boundaries of the configuration space or at infinity (cf. ibid.). In contraposition to Earman, this might be taken as an indication that a more sensible conceptualization of determinism involves not only the dynamical laws, but also boundary conditions. In fact, cases such as this may suggest that the distinction between laws and boundary or initial conditions may either not be principled, or, if principled, not very deep $9^{9}$ It should be noted that in Earman's own characterization of determinism for theories, which is captured by Definition 2, it only matters whether the theory in toto has the resources to uniquely evolve physically possible states of the systems under its purview. If the boundary conditions are part and parcel of the theory, I see no problem with this strategy. In order to sensibly count the boundary conditions as an integral part of the theory, however, some principled reasons for the particular stipulation that is pronounced need to be given. In other words, the choice among all the possible self-adjoint extensions must be made in the light of physically compelling arguments. In the absence of such arguments, no good case can be made for a particular stipulation and the boundary conditions cannot rightly be counted as part of the theory.

\footnotetext{
${ }^{9}$ There are more indications to this effect: E.g. the past hypothesis necessary to deduce the second law of thermodynamics from the time-reversal invariant equations of statistical mechanics is really nothing but an initial condition, of which many have assumed a law-like status, and in the case of space invaders (cf. Earman 1986), a natural way to evade the unnatural indeterminism is to impose boundary conditions at infinity. This latter case is exactly analogous to the sort of boundary conditions that arise in the quantum case discussed in the main text.
} 
There may well be a principled way of privileging a particular self-adjoint extension over the others. For some systems, there exists a uncountable infinity of (unitarily inequivalent) representations of the canonical commutation relations ${ }^{10}$ In these cases, the Hamiltonian of the system will not be essentially self-adjoint. The problem, however, is that without choosing a representation of the canonical commutation relations, a quantum theory of the system at stake can simply not be found. Thus, by formulating a quantum theory of the system, a particular self-adjoint extension is picked, the Hamiltonian thus fixed, and the indeterminism vanquished. Although all of this is true, the resolution is merely apparent, as Earman rightly notes: "[t] he indeterminacy in the dynamics has been passed on to the indeterminacy in the representation of the [canonical commutation relations]' (45). In other words, the problem hasn't been solved, but merely pushed back: unless we identify independent reasons to choose a particular, 'correct,' representation of the canonical commutation relations, the quantum indeterminism can only be avoided by an unprincipled fiat. I will not pursue this any further, but hasten to add that I know of no other reason to think that we will find compelling reasons for a particular stipulation in all physically possible cases of non-essentially self-adjoint Hamiltonians, be it via the choice of one among many (unitarily inequivalent) representations of the canonical commutation relations or not.

I will now leave the topic of Schrödinger evolution with this disappointingly indecisive conclusion. At the very least, however, this superficial survey of issues that arise in, and results that pertain to, the context of an investigation into whether the Schrödinger evolution, as folklore has it, is deterministic highlights how the question does not afford a simple and unqualified answer. For the remainder of this essay, I will, perhaps falsely, assume that the Schrödinger dynamics is perfectly deterministic for all physical systems of concern. Let us turn then to the other popular belief, viz. that the state vector reduction necessitated by measurements implies that quantum mechanics is irremediably indeterministic.

\subsection{The measurement problem}

Whether or not this is indeed the case depends crucially on the interpretation of quantum mechanics that one advocates ${ }^{11}$ An interpretation of quantum mechanics offers a solution to the measurement problem. The measurement problem can succinctly be stated as the inconsistency of the following three statements that seem to be endorsed or implied by the basic formalism of the theory (Maudlin 1995):

(1) The wave function $\psi$ completely describes the state of the physical system at stake.

(2) The linear Schrödinger equation always governs the dynamical evolution of the wave function of the system.

(3) Measurements of observables of the system have determinate outcomes.

That these three statements are inconsistent can easily be seen (ibid., 7f). Solving the measurement problem amounts to rejecting at least one of the above statements, thereby incurring the particular

\footnotetext{
${ }^{10}$ Cf. Earman 2009 (Sec. 10) for examples. Before any objections to the effect that the Stone-von Neumann theorem shows otherwise are aired, it should be noted that this theorem does not apply to these examples as they use a classical configuration space that is not $\mathbb{R}^{n}$. The relevant representation space is not $L_{\mathbb{C}}^{2}\left(\mathbb{R}^{n}, d^{n} x\right)$, but $L_{\mathbb{C}}^{2}\left(X^{n}, d \mu\right)$ with $d \mu$ a suitable measure on $X$.

${ }^{11}$ In what follows, I ignore instrumentalist interpretations. Presumably, if one is an instrumentalist, then one doesn't take quantum mechanics to have any bearing on whether the world is deterministic or not. I also ignore the Copenhagen interpretation because it doesn't offer a solution to the measurement problem which is still acceptable by contemporary standards.
} 
explanatory onus that is implied by denying that particular statement. This explanatory debt must be discharged by introducing 'new' physics. Interpretations of the first type, denying (1), must introduce additional variables that capture the additional degrees of freedom not encoded in $\psi$ and include a specification of their dynamics. These 'hidden-variables theories' may in principle be deterministic or indeterministic as long as they correctly reproduce the apparently stochastic behaviour of quantum systems. In practice, one of the major motivations to advocate a hiddenvariables theory, however, is to postulate an underlying, 'hidden,' reality that behaves perfectly deterministically. Bohmian mechanics, to be discussed in Section 2.3 , is the main representative of this camp.

Interpretations of the second type modify the Schrödinger dynamics by introducing a new dynamical regime that holds sway during 'measurements.' This modification must either give a principled way of distinguishing a privileged class of physical interactions ('measurements') and give the relevant dynamics for these interactions, or introduce a new non-linear dynamics altogether. The most prominent example in this camp is the so-called GRW theory formulated in Ghirardi et al. 1986. GRW takes the second route and postulates a fundamentally stochastic, and thus indeterministic, dynamics. As far as GRW is concerned, probabilities exist objectively in the world. I will briefly return to GRW in Section 3.2 .

Finally, the third type of interpretations are those theories denying that there are determinate measurement outcomes. On this account, 'new physics' must be introduced at least in the sense of offering an explanation as to why outcomes seem determinate when in fact they are not. Most prominently, this group comprises the Everettian many-worlds theories. These theories are fully deterministic insofar as they insist that the full dynamics is given by the Schrödinger equation 12 This essay will not venture into the thorny and hotly debated controversy regarding the possibility of coherently introducing probabilities in order to recreate the quantum mechanical statistics.

As can be seen from this menu of available (realist) interpretations, it is far from a foregone conclusion that quantum mechanics, appropriately extended or modified to solve the measurement problem, is indeterministic. While many of the caveats discssued in Section 2.1 were of a rather technical nature and may well turn out to play little physical role, the issue of how to solve the measurement problem, and thus of whether quantum mechanics requires indeterminism in solving it, cannot be skirted. I make no pretense of contributing to such a solution. The remainder of this section shall be dedicated to how the transcendence of determinism may resurface in quantum mechanics.

Given these different verdicts concerning determinism, transcendence would arise if two empirically adequate interpretations on opposing sides of the divide could be shown to be empirically equivalent. Such a tie would only be broken if both interpretations fall out of empirical favour. But the problem is insofar academic as the indeterministic GRW is inequivalent to both its deterministic competitors Bohmian mechanics and many worlds: its non-linear dynamics yields in principle measurable differences to either of the other two 13 That the deterministic Bohmian mechanics and many worlds are empirically equivalent doesn't entail transcendence since both theories are deterministic. The cleanest example of a pair of empirically equivalent interpretations which disagree concerning determinism is Bohmian and Nelsonian mechanics.

\footnotetext{
${ }^{12}$ More precisely, they are deterministic if the Schrödinger dynamics is. As we have seen in Sec. 2.1, this need not be the case.

${ }^{13}$ Cf. e.g. Albert 1992.
} 


\subsection{Transcendence again: Bohmian and Nelsonian mechanics}

Bohmian mechanics assumes that all elementary physical systems ('particles') have determinate positions, i.e., position plays a privileged role over all other observables ${ }^{14}$ Apart from the precisely positioned particles, the theory regards as fundamental the wave function and, if present, force fields. Consider a system of $N$ particles with masses $m_{1}, \ldots, m_{N}$ with actual positions $\mathbf{Q}_{1}, \ldots, \mathbf{Q}_{N}$ moving in physical space $\mathbb{R}^{3}$ with a wave function $\psi=\psi\left(\mathbf{q}_{1}, \ldots, \mathbf{q}_{N}\right)=: \psi(q)$ defined on the configuration space of the system. The state of the $N$-particle system is thus completely described by its wave function $\psi(q)$ on the space of possible configurations $q=\left(\mathbf{q}_{1}, \ldots, \mathbf{q}_{N}\right)$ and the actual configuration $Q$ determined by the actual positions $\left(\mathbf{Q}_{1}, \ldots, \mathbf{Q}_{N}\right)$. The dynamics of the wave function is given by the usual Schrödinger equation. The dynamics of the actual positions is given by a first-order evolution equation, Bohm's so-called guiding equation, for $Q(t)$ :

$$
\frac{d \mathbf{Q}_{k}(t)}{d t}=\left(\frac{\hbar}{m_{k}}\right) \operatorname{Im}\left[\frac{\nabla_{k} \psi}{\psi}\right]\left(\mathbf{Q}_{1}, \ldots, \mathbf{Q}_{N}\right),
$$

where $\nabla_{k}$ is the covariant derivative with respect to the coordinates of the $k$-th particle. Evidently, the dynamics of the actual positions of the particles depends on the wave function $\psi$. In this sense, the wave function 'guides' the particles along their trajectories, it 'pushes' them from one location to the next.

Every elementary presentation of Bohmian mechanics presents it as a deterministic theory. What is needed to establish this are robust existence and uniqueness theorems also for the guiding equation (1). If the wave function $\psi$ has nodes, then the denominator on the right-hand side of (1) vanishes, which complicates the establishment of such theorems. But it has been done (Berndl et al. 1995). In fact, it can be shown (Berndl 1996) that such theorems hold for a large class of potentials, including the standard cases such as the $N$-particle Coulomb interaction with arbitrary masses and charges. It is not the case, however, that the initial value problem always has a unique global solution: There are initial conditions of measure zero for which this is provably not the case (Berndl 1996, 80). Overall, however, a convincing argument can be made that under physically plausible constraints, Bohmian mechanics is a deterministic theory in the sense of Definition 2.

The transcendence issue that was raised in Section 1 resurfaces in the context of Bohmian mechanics. Bohmian mechanics is almost, but not quite, empirically equivalent to standard quantum mechanics ${ }^{15}$ As it turns out, however, there is an ineliminably stochastic and thus indeterministic rival theory that is empirically equivalent to Bohmian mechanics: Nelson's mechanics ${ }^{16}$ Nelson's mechanics is not only fundamentally indeterministic in that the dynamical equation for the elementary particles is stochastic, but it is also unusual in that it regards the wave function as merely derivative rather than fundamental, while all of the major realist solutions to the measurement problem take the wave function to be fundamental $\left[{ }^{17}\right.$ Nelson's programme has some major weaknesses and it is not clear whether it can be completed ${ }^{18}$ If it can, then we are again faced with the possibility that the question of determinism may transcend any possible experience. Note,

\footnotetext{
${ }^{14}$ Cf. also Timpson (this volume, Sec. 4). For the best accessible introduction to Bohmian mechanics that I am aware of, see Albert 1992 (Ch. 7). For another authoritative review, see Goldstein 2006.

${ }^{15}$ Cf. Albert 1992 (134); Timpson (this volume, Sec. 4).

${ }^{16}$ The standard reference is Nelson 1985, the classic paper is Nelson 1966. For a conceptual introduction, see Bacciagaluppi 2005, where the empirical equivalence between the two theories is explained in Sec. 2. I wish to thank Guido Bacciagaluppi for drawing my attention to Nelson's mechanics.

${ }^{17}$ Some authors regard Nelson's mechanics as analogous to Bohmian mechanics, with the wave function given, except for the Nelsonian guiding equation which is stochastic rather than deterministic (Bacciagaluppi, private communication).

${ }^{18}$ Bacciagaluppi 2005 (Secs. 4 and 5).
} 
however, that this transcendence is conditional in that it will only obtain if Bohm or Nelson win out. Should our data favour GRW or many worlds, then transcendence not only doesn't arise, but we will also have compelling reason to think that indeterminism or determinism is true of our actual world; unless, of course, someone comes along and formulates a consistent and empirically equivalent alternative to the winner theory whose verdict regarding determinism contradicts that of the winner.

\section{Alleged proofs of indeterminism}

The potential transcendence of determinism may equally be obliterated by a proof that the world, or the uniquely empirically adequate candidate for a fundamental theory to describe that world, is indeterministic. Interestingly, there exist such alleged proofs; both a theorem due to Andrew Gleason (1957) and the recently proposed and somewhat ambitiously termed 'strong free will theorem' have been interpreted to establish indeterminism from innocuous assumptions. But of course a proof is only as good as its premises. These premises may either be unduly strong, as is arguably the case for the strong free will theorem, or simply define a different game altogether, as it turns out for Gleason's theorem.

\subsection{Gleason's theorem}

When we turn to quantum mechanics, the first result that deserves to be mentioned in the context of determinism is Gleason's theorem 19 Like all other basic theorems of quantum mechanics, it does not in any way rely on an interpretation of the theory, i.e. it does not presuppose a solution to the measurement problem. Just as Bell's theorem and the Kochen-Specker theorem, it can thus be seen as imposing constraints on any viable interpretation. Thus, if it successfully proves indeterminism from premises that are accepted by all extant or even possible solutions to the measurement problem, the considerations in Section 2.3 will become obsolete.

What follows in the remainder of this section is by necessity more technical. Its main conclusion will be that that Gleason's theorem fails to rule out deterministic interpretations. The theorem can be stated as follows 20

Theorem 1 (Gleason 1957) For separable Hilbert spaces $\mathcal{H}$ of dimension greater than two, all probability measures $\mu$ over the set of all subspaces of $\mathcal{H}$ or, more specifically, over the 'projection lattice' of $\mathcal{H}$ are of the form $\mu\left(\hat{P}_{i}\right)=\operatorname{Tr}\left(\hat{P}_{i} \hat{W}\right)$, where $\hat{P}_{i}$ is the projector onto a ray in $\mathcal{H}, \hat{W}$ is the density operator describing the state of the system, and $\operatorname{Tr}$ is the trace.

In other words, the usual statistical algorithm of quantum mechanics, also known as the (generalized) Born rule, represents the only possibility to consistently assign probabilities to possible experimental outcomes, i.e. to experimental questions asked of a particular state - at least for Hilbert spaces of more than two dimensions. In attributing such a prominent, and indispensable, role to Born's rule, Gleason's theorem places strong constraints on any attempts to modify standard quantum mechanics in response to the measurement problem. In particular, it is thought to severely constrain the construction of hidden-variables theories. In fact, it is often taken to imply

\footnotetext{
${ }^{19}$ One may ask why I focus on Gleason's theorem rather than the Kochen-Specker theorem as the latter equally rules out deterministic, non-contextual hidden-variables theories in that it also rules out dispersion-free probability measures on the lattice of projections. Apart from the historical precedence, the choice is purely pedagogically motivated.

${ }^{20}$ Cf. e.g. Redhead 1987 (28) or Pitowsky (this volume, Sec. 3).
} 
that there cannot be a hidden-variables theory, at least not for $\operatorname{dim}(\mathcal{H})>2$. The ground for this is the ineliminable stochasticity that it demands of the basic formalism of quantum mechanics. If true, this would rule out deterministic theories such as Bohmian mechanics. A deterministic theory can be thought of as claiming a bivalent probability measure, i.e. one with a range $\{0,1\}$ rather than $[0,1]$, and thus determining, with certainty, what the measurement outcomes will be. ${ }^{21}$ The problem with this is that Gleason's theorem implies that there cannot be a bivalent probability measure. In fact, as Redhead puts it, 'Gleason's theorem assures us that discontinuous measures are not possible for the three-[and higher-]dimensional case' $(1987,28)$. Note that, if anything, this argument proceeding from Gleason prohibits deterministic hidden-variables theories, and certainly not hidden-variables theories tout court. This is an important qualification, since there are other alleged no-go theorems against hidden-variables theories that on closer inspection turn out only to preclude certain types of hidden-variables theories, but not all of them.

In fact, as John Bell (1966) was the first to note, it only disqualifies non-contextual deterministic hidden-variables theories. Since Bohmian mechanics, as noted above, is a contextual hiddenvariables theory, it remains unscathed by Gleason's theorem. A corollary of Gleason's theorem is that for Hilbert spaces of dimension greater than two, an additivity requirement for expectation values of commuting operators cannot be met by states with determinate hidden properties, i.e. determinate values for some hidden variables. Bell calls these 'dispersion free states' because in a hidden-variables approach, the usual quantum-mechanical states captured by the wave functions are merely statistical averages 'over better defined states for which individually the results would be quite determined' (1) ${ }^{22}$ This additivity requirement-Proposition 2 below-demands that for any state, the expectation value of an operator that is the sum of any two commuting Hermitian operators is equal to the sum of the expectation values of these two operators.

Let me sketch Bell's target, the alleged argument from Gleason's theorem to the impossibility of a deterministic hidden-variables theory. It turns out that the relevant corollary of Gleason's theorem-Proposition 2 below-does not only follow from Gleason's theorem, Bell shows, but also from a seemingly innocuous set of assumptions. To prove the corollary from these simple assumptions allows Bell to isolate the underlying tacit assumption that ought to be rejected by the hidden-variables theorist. Thus, it is not Gleason's theorem that is challenged, but an assumption underlying the derivation of the corollary.

For the purposes of Bell's argument, it suffices to consider a real-valued, three-dimensional Hilbert space $\mathcal{H}$ and projection operators onto the states $|\varphi\rangle \in \mathcal{H}$. These states can be thought of as being represented by pairs of antipodal points on the unit sphere in a three-dimensional Euclidean space $\mathbb{E}^{3}$. Let us consider a complete set of projectors that splits the identity, in this case a triple of projectors projecting onto three orthogonal states represented by the intersection points of three orthogonal lines through the origin and the unit sphere. Since the three projectors commute, the expectation values of the projectors onto orthogonal rays also sum to one. Call this Proposition 0. Since the eigenvalues of projection operators are zero or one and therefore the expectation value of a projector is a non-negative number in $[0,1]$, and since any two projectors onto orthogonal rays can be regarded as members of a triple of projectors splitting the identity, a first proposition follows:

Proposition 1 For a given state of a system whose Hilbert space is $\mathcal{H}(\operatorname{dim}(\mathcal{H})>2)$, if the

\footnotetext{
${ }^{21}$ Strictly speaking, these are conditional probabilities, i.e. the probability of a certain measurement outcome given the pre-measurement state of the system and the measurement apparatus.

${ }^{22}$ The stress on commuting operators occurs because Bell's discussion of Gleason's theorem follows his rejection of von Neumann's old no-go argument against hidden-variables theories that used the unacceptably strong premise that the expectation values for non-commuting operators must be additive.
} 
expectation value for a projector onto a particular ray is one for that state, then the expectation value for projectors onto any orthogonal ray is zero for the same state.

If we look at any two orthogonal rays and the two-dimensional subspace they span, then it follows from Proposition 0 that the sum of the expectation values must be the same for any pair of projectors onto the two orthogonal rays spanning this subspace. In particular, we have

Proposition 2 For a given state of a system with Hilbert space $\mathcal{H}(\operatorname{dim}(\mathcal{H})>2)$, if the expectation values for some pair of projectors onto orthogonal rays are both zero, then the expectation value for the projector onto any ray in the subspace spanned by the two original rays vanishes.

In a deterministic hidden-variables theory, the expectation values for projectors will be either 0 or 1 . It then follows from Proposition 0 that both these values must occur. Since no other values are possible, there must be pairs of arbitrarily close points on the unit sphere for which the expectation values for the projectors onto the corresponding rays would be 0 and 1 , respectively. But as Bell $(1966,7 f)$ shows, an easy one-page proof that repeatedly uses Propositions 1 and 2 can be given that establishes that points in such a pair must be a certain minimum distance from one another. Thus, we have the contradiction that implies that the expectation values for projectors onto rays cannot be bivalent in the sense of only taking the values 0 and 1 . Thus, Bell offers, from apparently innocuous assumptions, a direct proof of the corollary to Gleason's theorem.

The problem with this proof, as Bell argues, is that it too, just like von Neumann's alleged no-go proof of hidden-variables theories, makes an unreasonable demand on the candidate hiddenvariables theory. Reconsider Proposition 2, The projector onto $\left|\alpha \phi_{1}+\beta \phi_{2}\right\rangle$ only commutes with the projectors onto $\left|\phi_{1}\right\rangle$ and $\left|\phi_{2}\right\rangle$ if either $\alpha$ or $\beta$ is zero, i.e. if it doesn't project onto a ray in the two-dimensional subspace other than the two original rays. In general, therefore, a measurement of the projector onto $\left|\alpha \phi_{1}+\beta \phi_{2}\right\rangle$ cannot be made simultaneously to a measurement of either of the projectors onto $\left|\phi_{1}\right\rangle$ or $\left|\phi_{2}\right\rangle$. It will require a distinct experimental set-up. Proposition 2 thus non-trivially relates the outcomes of measurements that cannot be performed simultaneously. Although quantum-mechanical averages over the 'dispersion-free' states of a hidden-variables theory must conform to Proposition 2, there is no reason that the dispersion-free states themselves do. According to Bell, the tacit assumption underlying Proposition 2 that ought to be rejected by a hidden-variables theorist, and certainly is rejected in Bohmian mechanics, is that the measurement of an observable yields, by necessity, the same outcome independently of what other measurements are performed simultaneously. What this tacit assumption implies is that the projectors onto $\left|\phi_{3}\right\rangle$ and either onto $\left|\phi_{1}\right\rangle$ or $\left|\alpha \phi_{1}+\beta \phi_{2}\right\rangle$, where $\left|\phi_{1}\right\rangle$ and $\left|\alpha \phi_{1}+\beta \phi_{2}\right\rangle$ are each orthogonal to $\left|\phi_{3}\right\rangle$ but not to one another, i.e. $\alpha \neq 0$, could be measured simultaneously (pairwise) and that the outcomes for the measurements of the projector onto $\left|\phi_{3}\right\rangle$ is the same regardless of whether it is accompanied by a measurement of the projector onto $\left|\phi_{1}\right\rangle$ or $\left|\alpha \phi_{1}+\beta \phi_{2}\right\rangle$. But the two pairs of measurements cannot be made simultaneously and require a different experimental set-up. A hidden-variables theory needs not accept that in both cases, the outcomes for the measurement of the projector onto $\left|\phi_{3}\right\rangle$ are the same. In other words, a hidden-variables theorist can opt for contextualism, the rejection of this tacit assumption. Such contextuality may formally be captured in that different observables may be associated with one and the same operator such that which observable is in fact measured depends on the experimental context.

In Bohmian mechanics, since position is privileged in that it alone has determinate values, other observables are only contextually defined. This means, roughly, that the outcome of a measurement of a non-configurational (i.e., non-position) observable depends on the experimental context. For example, whether the measurement of $z$-spin on a particle in a superposition state of $z$-spin up and 
$z$-spin down actually results in 'up' or 'down' not only depends on the exact position of the particle (and the wave function), but also on the precise set-up of the measurement device ${ }^{23}$

Thus, Gleason's theorem only rules out non-contextual deterministic hidden-variables theories, but does not affect hidden-variables theories that accept contextualism, such as Bohmian mechanics. Consequently, Gleason's theorem fails to establish that any solution of the measurement problem must accept indeterminism and it might still be the case that, given the empirical success of quantum mechanics, probabilities may be objectively in the world, or they may merely reflect our ignorance of the exact 'dispersion-free' states of quantum-mechanical systems. Recently, however, a result has been published of which it was claimed that it establishes that nature herself is indeterministic.

\subsection{The (strong) free will theorem}

John Conway and Simon Kochen have recently published a new theorem which they have auspiciously titled '(strong) free will theorem.24 Conway and Kochen ask us to consider our typical EPR-Bohm experiment, this time with spin-1 particles of which we measure, in both wings of the experiment, the squared spin in some direction. These measurements will invariably produce outcomes 0 and 1. For this version of the EPR-Bohm experiment, quantum mechanics entails the following proposition, called 'SPIN,' to be used as premise for both the Kochen-Specker as well as the free will theorems:

Axiom 1 (SPIN) 'Measurements of the squared (components of) spin of a spin 1 particle in three orthogonal directions always give the answers 1, 0, 1 in some order.' (Conway and Kochen 2009, 227)

SPIN is of course implied by quantum mechanics since the squared spin operators $\hat{S}_{x}^{2}, \hat{S}_{y}^{2}, \hat{S}_{z}^{2}$ commute and always sum to two for a spin-1 particle. Conway and Kochen define 101 functions as functions from the set of triples of orthogonal directions to 1, 0, 1 in some order with the following properties: (i) they take the same values for pairs of opposite directions; and (ii) they never map two orthogonal directions to 0. For purposes of illustration, the reader is invited to think of 101 functions as an assignment of either one of two colours (e.g. blue and red) to all points on the unit sphere such that for any triple of points in orthogonal directions, two points are painted blue and one is painted red. It can now be shown that a colouring satisfying these constraints cannot cover the entire surface of the unit sphere. In other words, a spin-1 particle cannot simultaneously have determinate values of squared spin along every direction. In fact, a subset of 33 pairs of directions, or points on the surface of the unit sphere, suffices to establish a contradiction in assigning determinate values of squared spin (or colours) in accordance to SPIN. This is essentially the content of the Kochen-Specker theorem 25

Theorem 2 (Kochen-Specker 1967) 'There does not exist a 101 function for the 33 pairs of directions of [the so-called 'Peres configuration'].' (ibid.)

How the Peres configuration is constructed in detail is immaterial for present purposes. What is not immaterial are the consequences that a hidden-variables theory faces in the aftermath of the

\footnotetext{
${ }^{23}$ Cf. Albert 1992 (145-155) for a lucid account of this contextuality.

${ }^{24}$ Conway and Kochen 2006, 2009. I will follow the presentation in their more recent work where they strengthen the result. Unless otherwise noted, 'free will theorem' will henceforth refer to the strengthened version of 2009 . Cf. Redhead 1987 (Chs. 5, 6) for a commendable presentation of the Kochen-Specker set-up and Menon 2010 for a very recent presentation and discussion of the free will theorem.

${ }^{25}$ Kochen and Specker 1967; cf. also Held 2006.
} 
Kochen-Specker theorem. As Redhead (1987, Ch. 5) explicates, accepting the Kochen-Specker theorem means either rejecting realism about hidden variables or embracing a form of contextualism. The former - the orthodox choice - denies that all observables have determinate values, encoded in hidden variables, prior to measurement. The latter can come in different fashions. One can, for instance, opt for what Redhead termed ontological contextuality and give up the bijective correspondence between the set of self-adjoint operators and the set of observables. Bohmian mechanics opts for an enviromental contextuality where the measured value of the observable depends, inter alia, on the experimental set-up. Translated into the particular circumstances of our EPR-Bohm experiment, this means that the operator $\hat{S}_{i}^{2}$ measures different observables depending on which other measurements are made co-temporaneously. Thus, the operator $\hat{S}_{i}^{2}$ does not simply encode the observable 'squared spin along direction $i$ ', but an observable such as 'squared spin along direction $i$, given that the other two orthogonal directions along which measurements in the same wing are made are $j$ and $k$ and given that the apparatus in the other wing is set to measure the squared spin in direction $l$ ''

Conway and Kochen add two more premises to SPIN to prove their free will theorem: TWIN and MIN. Assuming that we put a pair of entangled spin-1 particles in a 'singleton' state of total spin zero, non-local correlations will obtain: If the devices in both wings are set to measure the squared spin in the same direction, then they will measure the same outcome. The second axiom assumes as much:

Axiom 2 (TWIN) 'For twinned spin 1 particles, suppose experimenter A performs a triple experiment of measuring the squared spin component of particle a in three orthogonal directions $x, y, z$, while experimenter $B$ measures the twinned particle $b$ in one direction, $w$. Then if $w$ happens to be in the same direction as one of $x, y, z$, experimenter $B$ 's measurement will necessarily yield the same answer as the corresponding measurement by A.' (ibid., 228)

In fact, $w$ will be one of the 33 directions in the Peres configuration, while $x, y, z$ will be one of 40 particular orthogonal triples consisting of 16 triples from the Peres configuration and 24 triples resulting from the completion of orthogonal pairs in this configuration. The third axiom consists of two independent claims reminiscent of the premises assumed to derive Bell's theorem: a relativistic locality assumption and the presupposition that the experimenters can freely choose their settings:

Axiom 3 (MIN) 'Assume that the experiments performed by $A$ and $B$ are space-like separated. Then experimenter $B$ can freely choose any one of the 33 particular directions $w$, and a's response is independent of this choice. Similarly and independently, A can freely choose any one of the 40 triples $x, y, z$, and b's response is independent of that choice.' (ibid.)

If the locality assumption in MIN wouldn't hold, then there would be a frame of reference with respect to which the measurement outcome in $A$ 's wing (' $a$ 's response') would be influenced by a future event- $B$ 's choice of setting in her wing. Since this would be unacceptable, the locality assumption must hold. The idea behind the free-choice-in-settings part of MIN is that the experimenters' free will naturally underwrites their free and independent choices of measurement directions. To say that $A$ 's choice of a triple of orthogonal directions $\langle x, y, z\rangle$ is free means that it is not a function of what has transpired at earlier times in any frame of reference. In other words, $A$ 's choice at the indicated point in Figure 1 must be independent of what obtains anywhere in the shaded spacetime region.

Premised on SPIN, TWIN, and MIN, the free will theorem can now be proven. Conway and Kochen announce it in confident terms: 


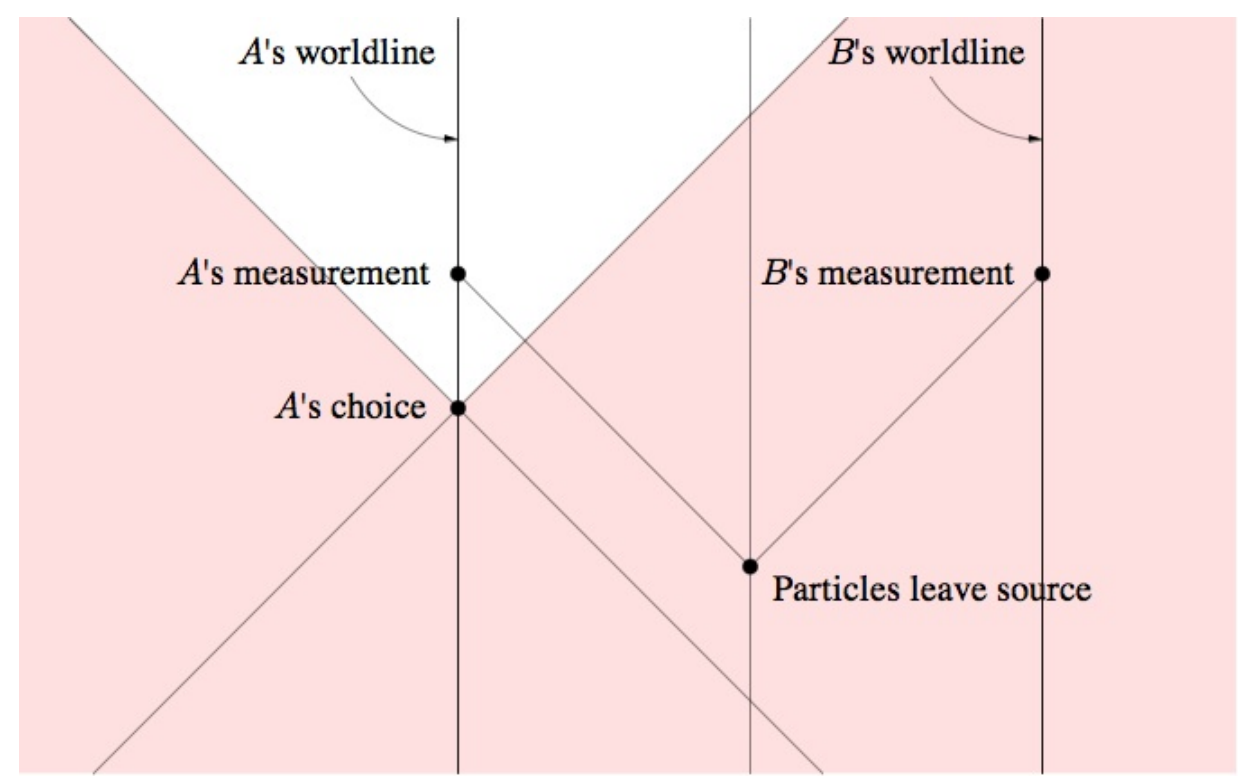

Figure 1: A's choice of settings must be independent of all events in the shaded region.

'The Free Will Theorem'... is the culmination of a series of theorems about quantum mechanics that began in the 1960s. It asserts, roughly, that if indeed we humans have free will, then elementary particles already have their own small share of this valuable commodity. (ibid., 226)

More specifically, they prove the following result:

Theorem 3 (Free will) 'The axioms SPIN, TWIN, and MIN imply that the response of a spin 1 particle to a triple experiment is free-that is to say, is not a function of properties of that part of the universe that is earlier than this response with respect to any given inertial frame.' (ibid., 228)

Essentially, if the experimenter's choice of measurement direction is free, then the particle's response to the measurement along the chosen direction is also free. More precisely, the particle's response is, as Conway and Kochen call it, 'semi-free,' since it still has to obey the constraint set by TWIN, i.e. it is really a pair of entangled particles that jointly give a free response to the measurement. Although I will not pursue this here, note that Conway and Kochen think that it is natural to suppose that [the particles'] freedom is the ultimate explanation of our own' (230) ${ }^{26}$ I will also not go through the details of the technical but rather straightforward proof of the theorem, as it does not offer additional illumination.

Does the free will theorem constrain potential solutions of the measurement problem? In particular, does it preclude hidden-variables theories? Undelayed by hesitation, Conway and Kochen offer a sweeping interpretation of their result:

Although... determinism may formally be shown to be consistent, there is no longer any evidence that supports it, in view of the fact that classical physics has been superseded by quantum mechanics, a non-deterministic theory. The import of the free will theorem is that it is not only current quantum theory, but the world itself that is non-deterministic, so that no future theory can return us to a clockwork universe. (ibid., 230; my emphasis)

\footnotetext{
${ }^{26}$ They also think that their result renders 'compatibalist' [sic!] approaches to the metaphysics of free will obsolete.
} 
If they are right in their unmitigated conclusion, then - from first principles - there simply can't be any deterministic theories, including Bohmian mechanics. But having re-emerged in the wake of both Gleason's and Kochen and Specker's theorems, Bohmian mechanics is a survivor. So let's see whether Theorem 3 indeed exterminates it.

SPIN and TWIN are entailed by quantum mechanics and thus much less vulnerable than MIN, which turns out to be both subtle and substantive. First, note that MIN consists really of four distinct assumptions, two assumptions of freedom of the experimenter's choice of measurement setting - one for each wing - and two assumptions of independence of the measurement outcomes of the settings in the other wing - again one for each wing. Since this latter is essentially a relativistic locality assumption that is denied in Bohmian mechanics, Theorem 3 does nothing towards establishing a robust no-go result against non-relativistic hidden-variables theories. What it offers, instead, is a proscription against relativistic hidden-variables theories. Conway and Kochen seem to recognize as much when they continue:

Granted our three axioms, the [free will theorem] shows that nature itself is nondeterministic. It follows that there can be no correct relativistic deterministic theory of nature. In particular, no relativistic version of a hidden variable theory such as Bohm's well-known theory... can exist. (ibid., 230f)

It has been claimed that this is not a new result, as it effectively reduces to Bell's theorem. Bell's theorem, experimentally well confirmed by the Aspect-Gisin experiments in Paris and Geneva. ${ }^{27}$ states that Bell's inequalities must be violated. This is usually interpreted to mean that the socalled Bell locality condition cannot hold. Bell locality can be unpacked as a conjunction of outcome and parameter independence. Outcome independence states that the measurement outcomes in one wing are probabilistically independent of the outcome in the other wing, given both measurement settings and the state of the bipartite system. Parameter independence states that the outcome in one wing is probabilistically independent of the setting chosen in the other wing, given the state. Goldstein et al. (forthcoming) read the independence half of MIN as essentially a version of parameter independence. Now, if determinism holds, the outcomes depend deterministically on the pre-measurement state of the bipartite system and both measurement settings. Thus, a deterministic theory trivially satisfies outcome independence. This means that a deterministic theory must violate parameter independence and, consequently, MIN. Conversely, this means that MIN rules out deterministic theories as an elementary consequence of Bell's theorem, without, as Tarun Menon (2010) puts it, "going through the rigmarole of Conway and Kochen's proof" (7).

Menon does not follow Goldstein et al. in their reading of MIN. He argues, quite convincingly, that Conway and Kochen do not conceive of the independence in MIN as a probabilistic independence as is usual in the entire literature. Instead, he shows that they must have some sort of counterfactual independence in mind. Thus, Theorem 3 at best rules out causal determinism, where the deterministic force flows from the dynamics. But as Section 1 made clear, determinism does not have to be underwritten by causation, and to assume counterfactual independence of the outcomes from the distant settings outstrips the justificatory potency of relativity. Concerning the independence claimed in MIN, Conway and Kochen thus find themselves between a rock and a hard place: either independence is interpreted statistically, and their theorem's novelty crumbles, or else it is interpreted causally, and the assumption becomes so strong that the Bohmian can part company with impunity.

There is a question whether Theorem 3 even rules out relativistic, contextual hidden-variables theories. The Bohmian can simply reject MIN, not on the basis of its relativistic locality assumption,

${ }^{27}$ Cf. Shimony (2009, Sec. 3). 
but because it postulates a freedom of the experimenter that a deterministic theory need not accept. MIN allows each experimenter's choice to be entirely unconstrained by the past history of the world. But in Bohmian mechanics, such a libertarian's paradise finds no place. In fact, in any theory that is deterministic in the sense of Definition 2, the experimenter's choice, like everything else, will be fixed given the totality of past events. In fact, as far as Theorem 3 is concerned, it's indeterminism in, indeterminism out.

This move is reminiscent of the objection originally made by Abner Shimony and collaborators (1976) to Bell's theory of local beables. Shimony et al. allege that Bell presupposes, in the derivation of a Bell-type inequality, 'spontaneous events, such as acts of free will of the experimenters' and this is illegitimate since indeterminism of the requisite sort, while possible, 'has not been proved and... may well be false' $(1976,99)$. Bell (1977) responds that in analyzing physical theories, it makes perfect sense to attribute such 'whim' to the experimenters, even though this 'superdeterminism' offers a possibility to evade Bell's theorem, avoid superluminal connections, and thus save local realism by postulating that both measurement outcomes and instrument settings are determined by variables in their common past, as Bell explicitly admits 28

Bell argues that it suffices to consider the freely chosen settings as being picked by a deterministic 'random' generator which is sufficiently sensitive to initial conditions that it appears to be producing perfectly random choices. He admits, though, that while it is plausible to assume that a deterministic 'random' generator permits sufficient freedom, and freedom of the right sort, to derive Bell's theorem, 'it might be that these reasonable ideas about physical randomizers are just wrong' $(1977,103)$. In this case, one may rationally argue that these superdeterministic conspiracies are 'more digestible' than the usual non-localities thought to follow from Bell's theorem. Bohmian mechanics is certainly compatible, not only with the resulting non-locality, but also with the possibility of a deterministic 'randomizer' in this sense.

Conway and Kochen grant that 'determinism, like solipsism, is logically possible' $(2006,1466)$. But determinism, in their view, is physically implausible at least, and perhaps physically impossible since ' $[\mathrm{p}]$ hysical induction, the primary tool of science, disappears if we are denied access to random samples' (ibid.). Menon (private communication) complains that indeterminism is certainly not necessary for our inductive practices or science entirely. Probability distributions, he points out, are routinely assigned to deterministic phase spaces. A sample, he claims in the vein of Bell (1977), is sufficiently random for scientific purposes if there fail to be correlations between the (appropriately coarse-grained) sample selection mechanism - the randomizer - and the set from which the sample is drawn. Again, Bohmian mechanics certainly satisfies this minimal condition for scientificity. Thus, Conway and Kochen, by demanding genuine randomness, and thus indeterminism, fall prey to the charge levelled against Bell by Shimony and collaborators; it is them who prejudge a metaphysical question in a priori ways - in fact the very metaphysical question they claim to have resolved by their theorem. Note, however, that the proof of their theorem assumes genuine indeterminism not permitted by a deterministic randomizer. Without the presupposed genuine indeterminism, their proof no longer goes through. Their case against indeterminism thus has all the virtues of theft over honest toil. It is truly indeterminism in, indeterminism out.

Somewhat orthogonal to the case they present against deterministic hidden-variables theories is their second argument, which goes against relativistic versions of GRW. In fact, they think that not only have they outlawed relativistic versions of GRW, but that the free will theorem implies 'that there can be no relativistic theory that provides a mechanism for reduction [of the wave function]' (ibid., 231). More specifically, Conway and Kochen attack the embryonic relativistic version of GRW called rGRWf that was recently proposed by Roderich Tumulka (2006). In a nutshell, Conway and

\footnotetext{
${ }^{28}$ For Bell's admission, see his $(1977,103 ; 1980,110)$.
} 
Kochen (2006) claim to encapsulate the stochastic element in rGRWf, viz. the randomly located and timed 'flashes,' in a sequence of random numbers generated prior to the measurements by some random mechanism or other. In the subsequent measurements, the particles would then simply 'look up' the response in the sequence of previously established outcomes. But in this case the responses of the particles would no longer be free as they would be a function of prior events (the sequence of random numbers), in violation of the free will theorem. Tumulka (2007) has responded that the statistics for the flashes cannot be pre-generated as the flash distribution depends also on external fields generated by the settings of the measurement instruments such that if it were pre-generated, the experimenters choices of the settings could not possibly be free. Conway and Kochen (2009) have shored up their original objection by proposing a scenario according to which the experimenters pre-generate 1320 sequences of random flash distributions in precise correspondence to the 1320 possible external fields resulting from the experimenters' 1320 distinct combined choices of settings. Thus, the information available to each particle would be a set of 1320 possible responses, out of which the particle 'looks up' the appropriate response given the freely chosen apparatus settings and the resulting external fields ${ }^{29}$ Again, the particle's response is a function of prior events, in violation of the free will theorem.

Conway and Kochen's argument against rGRWf does not succeed. Let us apply the pregenerating scheme for stochastic events they consider in their objection to Tumulka to their own case ${ }^{30}$ Thus, instead of the semi-freedom that the free will theorem concludes the particles command, let us establish the particle's responses prior to measurement by some sort of random, or quasi-random, mechanism. Let this mechanism produce as many lists of sequences of random responses that the particles may give as needed and let us impart that information on the particles as they leave the source for the two measurement wings. SPIN, TWIN, and MIN are all still valid, as in their argument against Tumulka. Since the particles later respond in accordance with these pre-generated lists by reading out the previously established outcome for the particular apparatus settings chosen by the experimenters, they violate the free will theorem as they are not functionally free from prior events. Thus, an application of their strategy against Tumulka to their own theorem exposes the frailty of that very theorem. Perhaps the problem this creates for Conway and Kochen is best appreciated if we think of this reverse-application of their strategy as striking them with a dilemma: either the strategy is legitimate, in which case they defeat themselves, or it is not a legitimate way of introducing chanciness into a theory, in which case Tumulka's rGRWf stands completely unaffected.

I prefer the second horn of the dilemma because it entails, correctly in my view, that the reformulation of a theory involving genuine stochasticity to capture the outcomes of measurements that Conway and Kochen propose does not leave the truly stochastic nature of the theory invariant. Thus, the original rGRWf (or another stochastic theory) and the one resulting from adding the pre-generation of the informational content of later stochastic events and somehow imparting that information on the quantum system cannot be equivalent: Originally, the free will theorem can be validly inferred from SPIN, TWIN, and MIN; in the reformulated theory, this is no longer the case. Hence, the theories cannot possibly be equivalent. This consideration clearly brings out the strangeness of Conway and Kochen's argument against rGRWf, a theory that, given its indeterminism, they should like. At least as far as their pre-generating scheme is concerned, they sit in the same boat as Tumulka. They should not sink it.

\footnotetext{
${ }^{29}$ It seems as if for the particle to be able to pick the right answer, it must 'know' the distant setting - in violation of MIN. Their argument thus derails, even if the following strike against it fails.

${ }^{30}$ I thank Guido Bacciagaluppi for suggesting to consider reverse-applying the scheme of Conway and Kochen to their own argument.
} 
It would be ironic if their argument against this preeminent version of GRW succeeded, as GRW is the only fully stochastic solution to the measurement problem. If it succeeded, we would have been left with deterministic solutions: hidden-variables theories and Everettian many-worlds theories. Under the presupposition that the Schrödinger evolution is deterministic for physically realistic systems, a solution to the measurement problem could only be indeterministic if it proceeded by denying that the Schrödinger evolution is the universally valid dynamical rule. Thus, at least if the measurement problem is understood as above, only a theory incorporating some reduction of the wave packet or 'collapse' could be indeterministic. If, counterfactually, their above argument for indeterminism succeeded, and if this second argument against GRW proved successful, then they would have struck down all known realist solutions to the measurement problem. In other words, they would have established that there is even no known candidate solution of the measurement problem. If borne out, that would be disappointing news indeed.

\section{Conclusions}

If you, honourable reader, leave this essay with the correct impression that the determinism question is a subtle matter that is far from decided, I shall be content. Insisting that it is currently wide open does not, however, amount to claiming that the issue forever transcends our epistemic limitations. While it may ultimately be beyond our grasp to resolve it, neither Suppes nor a similar argument based on the empirical equivalence of Bohmian and Nelsonian mechanics succeeds in establishing as much. Moving to quantum mechanics, I tried to dispel, or at least qualify, the orthodox views that the Schrödinger evolution is completely and safely deterministic and that the measurement process is ineliminably indeterministic. The former is only roughly true, while the latter depends entirely on a resolution of the measurement problem. Finally, two alleged proofs that nature must be indeterministic, relying on Gleason's and the recently proclaimed free will theorems, have been shown to be wanting. Let me be clear: The proofs of these theorems are perfectly valid; it's just that they fail to expel deterministic theories from being serious contenders in fundamental physics. After over two centuries and a quarter, Kant's Third Antinomy is still alive and kicking.

\section{Acknowledgements}

I am obliged to Guido Bacciagaluppi, Craig Callender, Tarun Menon, and Charlotte Werndl for discussions and comments on earlier drafts of this paper, and to the editors for their kind invitation and for their patience. This work has been partially supported by the Hellman Foundation at UCSD, which is gratefully acknowledged.

\section{References}

[1] Albert, D.Z. (1992), Quantum Mechanics and Experience (Harvard University Press).

[2] Bacciagaluppi, G. (2005), 'A conceptual introduction to Nelson's mechanics', in R. Buccheri, M. Saniga and A. Elitzur (eds.), Endophysics, Time, Quantum and the Subjective (World Scientific), 367-388.

[3] Bell, J.S. (1966), 'On the problem of hidden variables in quantum mechanics', Reviews of Modern Physics 38: 447-52; reprinted in his (1987), Speakable and Unspeakable in Quantum Mechanics (Cambridge University Press), 1-13. Page references are to the reprint. 
[4] Bell, J.S. (1977), 'Free variables and local causality', Lettres épistémologiques 15; reprinted in Dialectica 39 (1985): 103-106, and in his (1987) Speakable and Unspeakable in Quantum Mechanics (Cambridge University Press), 100-104. Page references are to the second reprint.

[5] Bell, J.S. (1980), 'Atomic-cascade photons and quantum-mechanical nonlocality', Comments on Atomic and Molecular Physics 9: 121-126; reprinted in his (1987), Speakable and Unspeakable in Quantum Mechanics (Cambridge University Press), 105-110. Page references are to the reprint.

[6] Berndl, K. (1996), 'Global existence and uniqueness of Bohmian mechanics', in J.T. Cushing, A. Fine and S. Goldstein (eds.), Bohmian Mechanics and Quantum Theory: An Appraisal (Kluwer), 77-86.

[7] Berndl, K., Dürr, D., Goldstein, S., Peruzzi, P. and N. Zanghì (1995), 'On the global existence of Bohmian mechanics', Communications in Mathematical Physics 173: 647-673.

[8] Conway, J.H. and Kochen, S. (2006), 'The free will theorem', Foundations of Physics 36: 1441-1473.

[9] Conway, J.H. and Kochen, S. (2009), 'The strong free will theorem', Notices of the American Mathematical Society 56: 226-232.

[10] Earman, J. (1986), A Primer on Determinism (Reidel).

[11] Earman, J. (2004), 'Determinism: What we have learned and what we still don't know', in J.K. Campbell et al. (eds.), Freedom and Determinism (MIT Press), 21-46.

[12] Earman, J. (2007), 'Aspects of determinism in modern physics', in J. Butterfield and J. Earman (eds.), Handbook in the Philosophy of Physics (North Holland), 1369-1434.

[13] Earman, J. (2008), 'How determinism can fail in classical physics and how quantum physics can (sometimes) provide a cure', Philosophy of Science 75: 817-829.

[14] Earman, J. (2009), 'Essential self-adjointness: implications for determinism and the classicalquantum correspondence', Synthese 169: 27-50.

[15] Ghirardi, G.C., Rimini, A. and Weber, T. (1986), 'Unified dynamics for microscopic and macroscopic systems', Physical Review D34: 470-491.

[16] Gleason, A.M. (1957), 'Measures on the closed subspaces of a Hilbert space', Journal of Mathematics and Mechanics 6: 885-893.

[17] Goldstein, S. (2006), 'Bohmian mechanics', in E. Zalta (ed.), Stanford Encyclopedia of Philosophy, http://plato.stanford.edu/entries/qm-bohm/.

[18] Goldstein, S., Tausk, D.V., Tumulka, R., and Zanghì, N. (forthcoming), 'What does the free will theorem actually prove?' to appear in Notices of the American Mathematical Society. Also available at http://arxiv.org/pdf/0905.4641v1.

[19] Held, C. (2006), 'The Kochen-Specker theorem', in E. Zalta (ed.), Stanford Encyclopedia of Philosophy, http://plato.stanford.edu/entries/kochen-specker/.

[20] Hoefer, C. (2003), 'Causal determinism', in E. Zalta (ed.), Stanford Encyclopedia of Philosophy, http://plato.stanford.edu/entries/determinism-causal/. 
[21] Kant, I. (1781/7 [1999]), Critique of Pure Reason (Cambridge University Press), translation by P. Guyer and A. Wood.

[22] Kochen, S. and Specker, E. (1967), 'The problem of hidden variables in quantum mechanics', Journal of Mathematics and Mechanics 17: 59-87.

[23] Loewer, B. (2001), 'Determinism and chance', Studies in the History and Philosophy of Modern Physics 32: 609-620.

[24] Maudlin, T. (1995), 'Three measurement problems', Topoi 14: 7-15.

[25] Menon, T. (2010), 'The Conway-Kochen free will theorem', manuscript.

[26] Nelson, E. (1966), 'Derivation of the Schrödinger equation from Newtonian mechanics', Physical Review 150: 1079-1085.

[27] Nelson, E. (1985), Quantum Fluctuations (Princeton University Press).

[28] Norton, J.D. (1999), 'A quantum mechanical supertask', Foundations of Physics 29: 1265-1302.

[29] Norton, J.D. (2008), 'The dome: An unexpectedly simply failure of determinism', Philosophy of Science 75: 786-798.

[30] Redhead, M. (1987), Incompleteness, Nonlocality, and Realism (Clarendon Press).

[31] Shimony, A. (2009), 'Bell's theorem', in E. Zalta (ed.), Stanford Encyclopedia of Philosophy, http://plato.stanford.edu/entries/bell-theorem/.

[32] Shimony, A., Horne, M.A. and Clauser, J.F. (1976), 'Comment on "The theory of local beables" ', Lettres épistémologiques 13; reprinted in Dialectica 39 (1985): 97-102.

[33] Suppes, P. (1993), 'The transcendental character of determinism', Midwest Studies in Philosophy 18: 242-257.

[34] Tumulka, R. (2006), 'A relativistic version of the Ghirardi-Rimini-Weber model', Journal of Statistical Physics 125: 821-840.

[35] Tumulka, R. (2007), 'Comment on "The free will theorem" ', Foundations of Physics 37: 186-197.

[36] Werndl, C. (2009), 'Are deterministic descriptions and indeterministic descriptions observationally equivalent?', Studies in the History and Philosophy of Modern Physics 40: 232-242.

[37] Winnie, J.A. (1997), 'Deterministic chaos and the nature of chance', in J. Earman and J.D. Norton (eds.), The Cosmos of Science: Essays of Exploration (University of Pittsburgh Press), 299-324.

[38] Wüthrich, C. (2006), Approaching the Planck Scale from a Generally Relativistic Point of View: A Philosophical Appraisal of Loop Quantum Gravity, PhD dissertation, University of Pittsburgh. 